\title{
Hypothalamic Obesity
}

\section{The Autonomic Hypothesis and the Lateral Hypothalamus}

\author{
G. A. Bray ${ }^{1}$, S. Inoue ${ }^{2}$, and Y. Nishizawa ${ }^{3}$ \\ ${ }^{1}$ Department of Medicine, Harbor-UCLA Medical Center, Torrance, California, USA; and ${ }^{2}$ The Second Department of Internal Medicine \\ University of Tokyo, Hongo, Tokyo, and ${ }^{3}$ The Second Department of Internal Medicine Osaka City Medical School, Osaka, Japan
}

Summary. Several lines of evidence support the hypothesis that derangements in the function of the autonomic nervous system play an important role in the development of hypothalamic obesity. Vagotomy below the diaphragm reverses the syndrome. In diabetic rats cured of their diabetes with transplants of fetal pancreatic tissue beneath the renal capsule, ventromedial hypothalamic (VMH) lesions do not produce the characteristic rise in food intake nor do they significantly increase serum insulin. These observations indicate that the hyperinsulinaemia following VMH lesions is the result of neural connections rather than from a circulating humoral factor released following $\mathrm{VMH}$ injury. The smaller salivary glands, reduced level of glucagon and impaired mobilization of fatty acids during stress in VMH lesioned rats point to reduced activity of the sympathetic nervous system. The impaired mobilization of fat from retroperitoneal depots in VMH lesioned rats during fasting is similar to the effect of sympathetic denervation of the retroperitoneal fat pad. Similarly, unilateral sympathectomy caused an increased weight gain in rats almost as much as unilateral VMH lesions but significantly less than in bilaterally lesioned rats. These studies with fasting and feeding implicate the VMH in the control of the sympathetic nervous system. When atropine and epinephrine were given to $\mathrm{VMH}$ lesioned rats, there was a significant depression in basal and glucose-stimulated levels of insulin. Finally when VMH lesions were placed after lateral hypothalamic lesions, the effect of the VMH lesions did not seem to be reduced, suggesting that the two effects are independent. A model dealing with the effects of VMH lesions is presented in an attempt to integrate these findings.
Key words: Sympathectomy, unilateral hypothalamic lesions, insulin, glucagon, body fat, food intake, atropine, epinephrine.

The syndrome of ventromedial hypothalamic obesity is produced by destruction of the ventromedial region of the hypothalamus. This syndrome has been recognized for more than 75 years [5]. A major milestone occurred when it was produced experimentally by Hetherington and Ranson in 1940 (16). The pathogenesis of this obesity differs from other models of obesity in which the obesity is genetically transmitted [5] or results from dietary manipulations $[22,26]$. Hypothalamic obesity and hyperphagia are associated with a number of changes of which both hyperphagia and increased concentrations of insulin occur as early events [19]. One formulation for this syndrome proposes that neurally mediated hyperinsulinaemia is the primary factor in the development of hypothalamic obesity with hyperinsulinaemia contributing to excess fat accumulation [23].

\section{The Development of Hypothalamic Obesity}

Early studies [5-7] considered hypothalamic obesity to result from the destruction of a 'satiety center' in the ventromedial hypothalamus (VMH). Ablation of this 'satiety center' was believed to remove the inhibitory influences to a 'hunger center' in the lateral hypothalamus (LH) and allow excess feeding. This intake of excessive amounts of food energy made the animals obese [7]. 
Recently several investigators have suggested that this interpretation may be overly simplistic. Han and Liu demonstrated that VMH-lesioned adult rats manifest obesity even if hyperphagia is prevented by tube-feeding [13]. Frohman and Bernardis showed that $\mathrm{VMH}$-lesioned weanling rats remained normophagic yet became obese [8].

When increased concentrations of insulin were first reported in VMH obesity, the hyperinsulinaemia was presumed to be secondary to hyperphagia and obesity since the hyperinsulinaemia disappeared after fasting [11]. However restricted food intake or pair-feeding failed to eliminate the increased concentrations of insulin [13, 17]. Moreover hyperinsulinaemia occurs within minutes or in the first few days after ventromedial hypothalamic lesions even

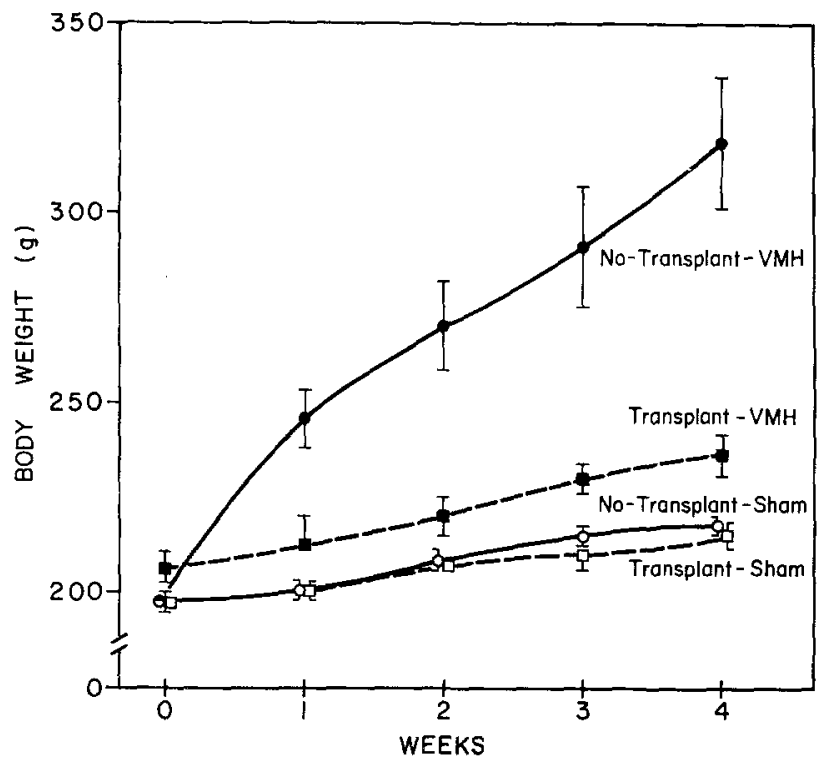

Fig. 1. Body weight of pancreatic transplanted $(\boldsymbol{\square}, \square)$ and intact rats $(\boldsymbol{\bullet}, 0)$. Body weight was followed for 4 weeks after VMH lesions $(O, \square$, sham-operated). The increase in the lesioned rats without pancreatic transplants was stastically significant. The rats with pancreatic transplants and VMH lesions gained a small amount of weight, but not significantly more than the sham-transplanted animals. All data are mean \pm SEM without hyperphagia or obesity $[17,31,38]$. Furthermore, York and Bray [43] and Goldman et al. [10] demonstrated that when $\beta$-cells of the pancreas were destroyed beforehand with streptozotocin to prevent the increase of insulin, obesity and hyperphagia were prevented or markedly attenuated.

Since insulin treatment can produce hyperphagia and obesity, attention has gradually shifted to hyperinsulinaemia as a central event in this syndrome. Until recently, however, the question remained unsettled as to whether hyperphagia or hyperinsulinaemia was the primary factor in the development of hypothalamic obesity. The mechanism for the increase of insulin in hypothalamic obesity also remained unsettled. It is clearly not secondary to the hyperphagia or obesity because hyperinsulinaemia occurs even when the food intake is limited and the increase of body weight is prevented $[13,17,31]$.

Two hypotheses have been advanced. One is humoral, postulating that an 'hypothalamic factor' which stimulated or suppresses pancreatic $\beta$-cells is released into the circulation from the hypothalamus and thus increases the release of insulin [28, 33]. Beta-endorphin could be such a factor [29]. The other hypothesis proposes 'neural mediation' which implies that stimuli for increased release of insulin by the $\beta$-cells occurs through the autonomic nervous system [1].

To separate these two possibilities, we performed the following experiment. Pancreatic $\beta$-cells were destroyed in Lewis rats by treatment with streptozotocin. Subsequently several fetal pancreases were transplanted underneath the renal capsule. In this way we could have rats with denervated pancreatic tissue. After recovery from diabetes, VMH lesions were performed and the rats were observed for 4 weeks [20, 21]. VMH-lesioned rats with an intact pancreatic tissue gained weight rapidly after VMH lesions, weighing $120 \mathrm{~g}$ more in 4 weeks (Fig. 1). VMH-lesioned rats with pancreatic transplants gained only $30 \mathrm{~g}$ but this increase was not significantly higher than the non-lesioned control

Table 1. Effects of VMH lesions on body fatness and food intake

\begin{tabular}{llcc}
\hline Group & Body density ${ }^{2}$ & Lee index ${ }^{\mathrm{a}}$ & $\begin{array}{c}\text { Food intake }^{b} \\
(\mathrm{~g} / \text { day) }\end{array}$ \\
\hline VMH-lesion intact pancreas & $1.0253 \pm 0.0167$ & $0.327 \pm 0.004$ & $29.8 \pm 0.8$ \\
Sham-lesion intact pancreas & $1.0770 \pm 0.0030$ & $0.288 \pm 0.002$ & $17.1 \pm 0.4$ \\
VMH-lesion transplanted pancreas & $1.0654 \pm 0.0116$ & $0.296 \pm 0.002$ & $20.5 \pm 0.5$ \\
Sham-lesion transplanted pancreas & $1.0794 \pm 0.0046$ & $0.292 \pm 0.003$ & $16.7 \pm 0.4$ \\
\hline
\end{tabular}
Value are means \pm SE. Lee index $=\sqrt[3]{\text { body wt }}(\mathrm{g}) /$ length $(\mathrm{cm}) . \quad{ }^{a}$ Data were obtained 4 weeks after VMH lesions; $\quad$ Data were
obtained during 2 nd week 
groups. Body fatness was compared by the methods of body density and Lee Index. VMH-lesioned rats with an intact pancreas were fatter than non-lesioned control rats in both comparisons, whereas VMH lesioned rats with pancreatic transplants showed no differences from control (Table 1).

Food intake of VMH lesioned rats with an intact pancreas increased about $70 \%$ whereas $\mathrm{VMH}$ lesioned rats with pancreatic transplants increased only about $20 \%$ (Table 1 ).

Serum insulin levels of VMH lesioned rats with an intact pancreas were about 3 times higher than in non-lesioned control rats with intact pancreatic tissue, whereas VMH lesioned rats with pancreatic transplants showed no differences from the unlesioned rats with pancreatic transplants (Fig. 2). The differences in serum insulin levels between control rats with an intact pancreas and those with pancreatic transplants may be explained by the fact that insulin is released into circulation and does not traverse the liver in the rats with pancreatic transplants [41].

If a humoral factor were the stimulus to pancreatic $\beta$-cells, this factor should reach the pancreatic tissue underneath the renal capsule and should have increased insulin release. Our results supported the hypothesis that the hyperinsulinaemia of hypothalamic obesity is primarily under neural control.

\section{Mechanism for the Hyperinsulinemia in Hypothalamic Obesity}

Both the sympathetic and parasympathetic nervous system appear to be involved in the hyperinsulinaemia of VMH-lesioned rats. Evidence for vagal hyperactivity first came from measurement of gastric acid. Ridley and Brooks demonstrated that VMH lesioned rats had gastric hyperacidity, a phenomenon related to vagal overactivity [37]. This result was subsequently confirmed by Powley and Opsahl [36] and by Inoue and Bray [18[. More direct evidence came from studies on vagotomy. In 1974 Powley and Opsahl demonstrated that interruption of the vagus nerves below the diaphragm could reverse the obesity which follows VMH lesions [36]. They suggested that hypersecretion of insulin was reduced after vagotomy and that reversal of obesity occurred because the hyperinsulinaemia was removed, but they did not measure serum insulin concentrations. Inoue and Bray [18] repeated this study and suggested that the reversal of body weight after vagotomy was in part due to restriction of food intake secondary to slowed gastric emptying. In this experiment a group of VMH-lesioned rats without vagotomy were pair-fed

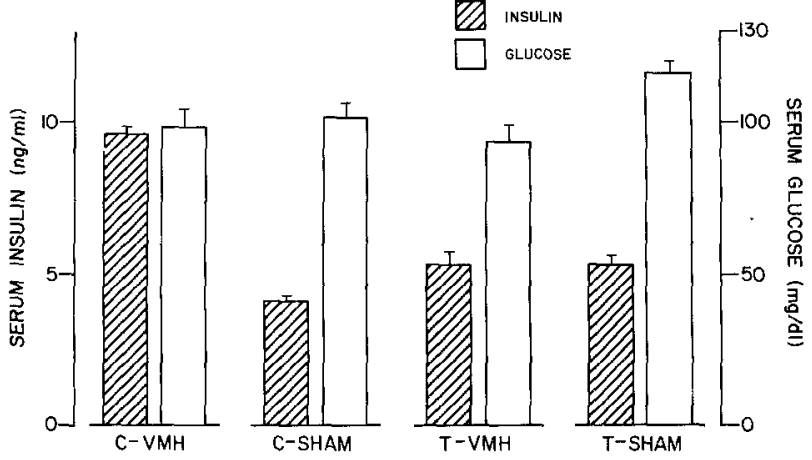

Fig. 2. Serum insulin and blood glucose levels in VMH lesioned and sham-operated rats 4 weeks after operation. Each column is plotted as mean $\pm \mathrm{SEM}$. $\mathrm{C}=$ intact pancreas; $\mathrm{T}=$ pancreatic transplants

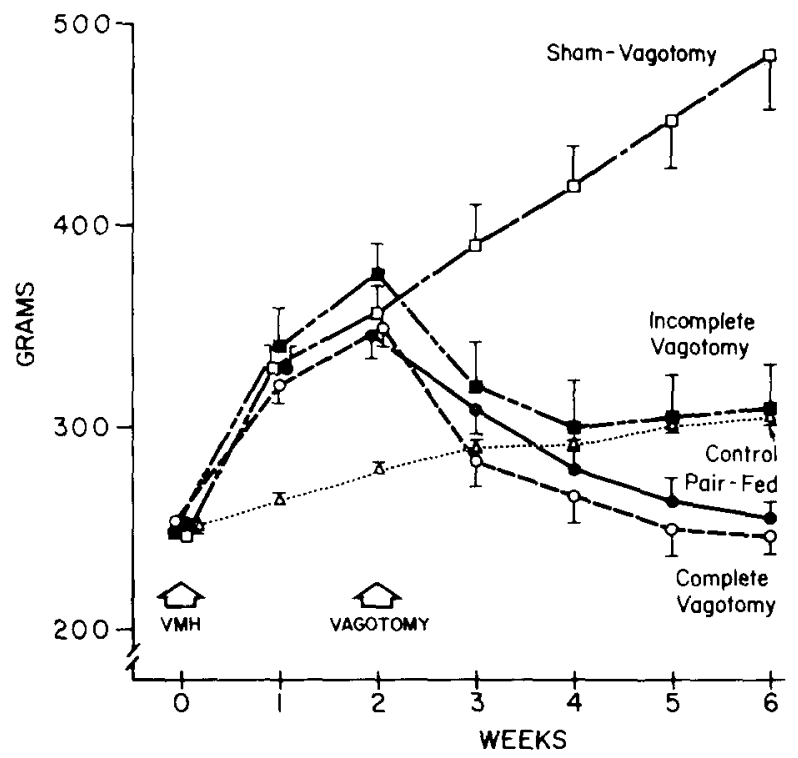

Fig. 3. Body weight of VMH lesioned and sham-lesioned vagotomized rats. Each group is plotted as mean \pm SEM. VMH with sham-vagotomy $=-\square-$; $\mathrm{VMH}$ with incomplete vagotomy $=$ - - ; VMH with complete

vagotomy $=-0^{-}$; VMH with sham-vagotomy and pair-fed to complete vagotomy $=-\mathbf{-}$; and control group $-\Delta-$

to the VMH lesioned rats with complete vagotomy [18]. As shown in Figure 3, VMH-lesioned rats with complete vagotomy lost weight rapidly during the first two weeks but stopped losing weight at the third week. VMH-lesioned rats without vagotomy which were pair-fed to those with complete vagotomy also showed a decline in body weight similar to the group with complete vagotomy. There was no difference in body weight at any time between these two groups.

The Lee index of obesity and serum insulin levels also showed no difference between these two groups 
Table 2. Effect of vagotomy, Lee index, food intake and serum insulin levels of VMH-lesioned rats

\begin{tabular}{|c|c|c|c|}
\hline Group & Lee inde $x^{a}$ & $\begin{array}{l}\text { Food intake } \\
\text { (g/day) }\end{array}$ & $\begin{array}{l}\text { Serum insulin } \\
(\mathrm{ng} / \mathrm{ml})\end{array}$ \\
\hline 1. VMH-lesion sham vagotomy & $0.352 \pm 0.011$ & $32.0 \pm 1.3$ & $11.1 \pm 1.6$ \\
\hline 2a. VMH-lesion complete vagotomy & $0.292 \pm 0.002$ & $8.7 \pm 1.6$ & $2.6 \pm 0.2$ \\
\hline 2b. VMH-lesion incomplete vagotomy & $0.314 \pm 0.010$ & $18.4 \pm 4.5$ & $5.0 \pm 0.1$ \\
\hline 3. VMH-lesion pair-fed to $2 \mathrm{a}$ & $0.293 \pm 0.005$ & $8.7 \pm 0.1$ & $3.3 \pm 0.5$ \\
\hline 4. Sham operations & $0.300 \pm 0.003$ & $19.2 \pm 0.7$ & $2.9 \pm 0.5$ \\
\hline
\end{tabular}

Values are means $\pm \mathrm{SE}$. ${ }^{a}$ Lee index $=3 \sqrt{\text { body } w t}(\mathrm{~g}) /$ length $(\mathrm{cm}) . \quad{ }^{\mathrm{b}}$ Data were obtained in serum after overnight fast performed 4 weeks after vagotomy or sham operated $\sqrt{ }$

Table 3. Effect of diet and $\mathrm{VMH}$ injury on metabolic parameters and weight of the salivary glands ${ }^{\mathrm{a}}$

\begin{tabular}{|c|c|c|c|c|c|c|c|}
\hline Diet & Operation & $\mathrm{n}$ & $\begin{array}{l}\text { Body } \\
\text { weight } \\
\mathrm{g}\end{array}$ & $\begin{array}{l}\text { Salivary } \\
\text { glands } \\
\text { mg }\end{array}$ & $\begin{array}{l}\text { Plasma } \\
\text { Triglyceride } \\
\mathrm{mg} / \mathrm{dl}\end{array}$ & $\begin{array}{l}\text { Insulin } \\
\mathrm{ng} / \mathrm{ml}\end{array}$ & $\begin{array}{l}\text { Glucagon } \\
\mathrm{pg} / \mathrm{ml}\end{array}$ \\
\hline Low fat & Sham & 6 & $296 \pm 4$ & $495 \pm 17$ & $24 \pm 4$ & $1.2 \pm 0.2$ & $357 \pm 37$ \\
\hline Low fat & VMH & 6 & $435 \pm 18$ & $450 \pm 4$ & $79 \pm 21$ & $2.4 \pm 0.3$ & $212 \pm 19$ \\
\hline High fat & Sham & 6 & $366 \pm 8$ & $549 \pm 10$ & $23 \pm 9$ & $0.9 \pm 0.1$ & $300 \pm 38$ \\
\hline High fat & VMH & 6 & $645 \pm 16$ & $478 \pm 22$ & $45 \pm 7$ & $3.7 \pm 1.0$ & $241 \pm 21$ \\
\hline
\end{tabular}

a Data were obtained in rats 10 weeks following VMH lesions or sham-operation

(Table 2). Food intake in the group with complete vagotomy showed a remarkable decrease during the experiment. Even at the fourth week their food intake was less than one-third of that found in the VMH-lesioned rats with vagotomy, and was about half the amount eaten by normal rats (Table 2).

Thus it was concluded that subdiaphragmatic vagotomy reverses obesity primarily by reducing food intake due to reduced gastric emptying and not by inhibiting insulin release. Nevertheless, we found a positive correlation between basal serum insulin and basal gastric acidity in VMH lesioned rats $(r=0.70$, $\mathrm{P}<0.01$ ) [18]. Recently Berthoud and Jeanrenaud reported that acute hyperinsulinaemia which started within an hour after VMH lesions could be reversed by acute vagotomy [2]. When the vagus nerve is sectioned before $\mathrm{VMH}$ lesions are made, the weight gain is attenuated indicating that the order of the procedures is important. These findings strongly suggest that the vagus plays an important role in the mechanism of hyperinsulinaemia in rats with $\mathrm{VMH}$ lesions $[1,42]$.

The sympathetic nervous system also plays a role in the hyperinsulinaemia associated with the syndrome of hypothalamic obesity, but the relative importance of these two components of the autonomic nervous system remains unclear. Support for the concept that VMH lesions reduce the activity of the sympathetic nervous system was first obtained by finding smaller submaxillary salivary glands and reduced serum glucagon levels in rats with VMHlesions [22]. Some of these data are presented in Table 3. The pair-feeding used in this experiment to keep food intake at comparable levels did not correct the glucagon levels or keep the salivary glands at normal weight [22].

To further explore the effects of VMH lesions on the function of the sympathetic nervous system, two groups of experiments were performed. In the first, the changes in non-esterified fatty acids (NEFA) and glycerol in the serum were used as indices for mobilization of lipid from adipose tissue stores. In this protocol VMH lesioned rats were allowed to gain weight for 5 days. At this time their food was restricted and the body weight returned to the same level as the sham-operated animals. After an additional 7 days one of several experiments was performed [34], including the one with 2-deoxy-D-glucose (2-DG) shown in Figure 4. The animals for this experiment had been adrenodemedullated previously. On the experimental day, a dose of 2-DG of $500 \mathrm{mg} / \mathrm{kg}$ was given IP and the animals decapitated 30 min later. The concentration of NEFA and glycerol rose significantly less in the VMH-lesioned rats than in the lean controls. Adrenodemedullation did not modify the effect of 2-deoxy-D-glucose on the rise of NEFA or glycerol, but did partially block the rise in glucose. The levels of insulin were significantly higher in the 

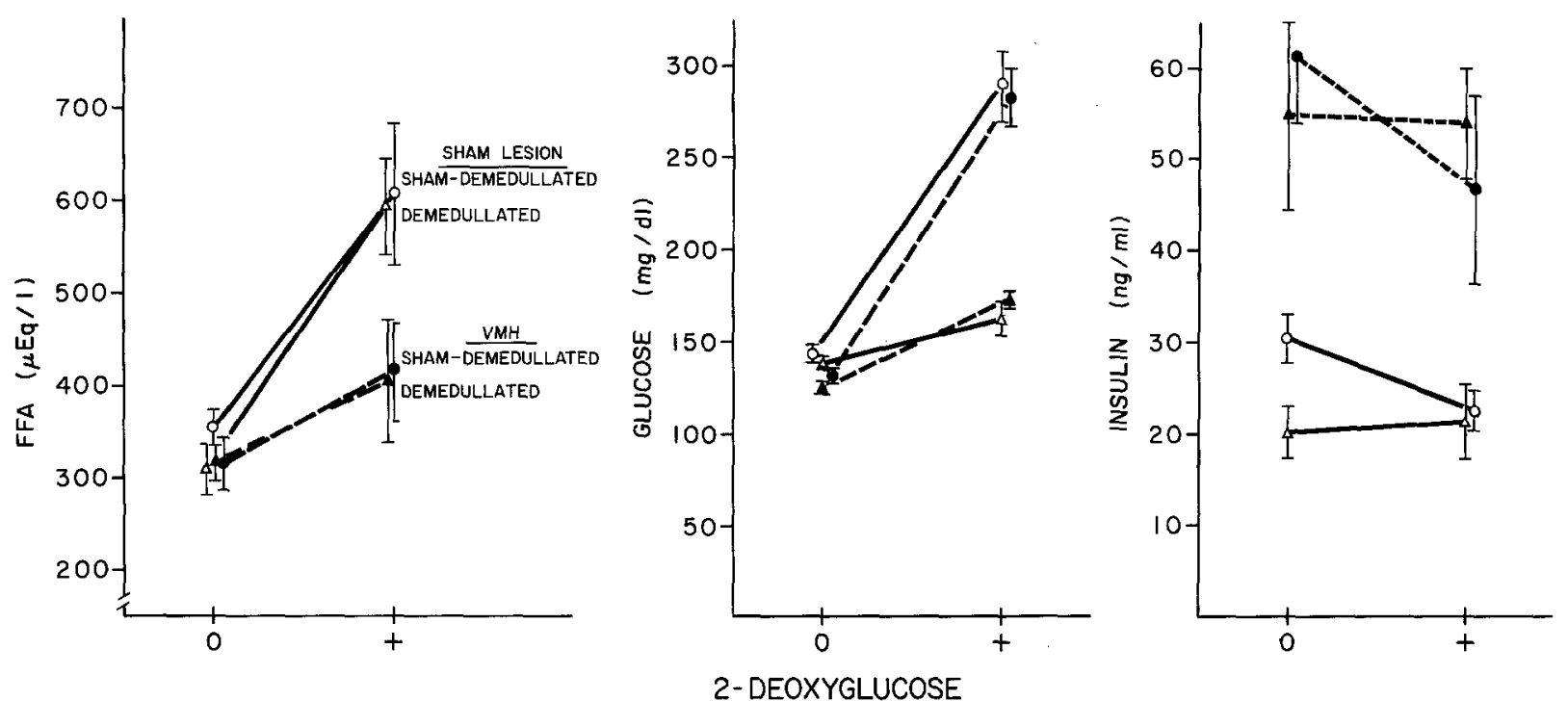

Fig. 4. Effect of 2-deoxyglucose on free fatty acids, glucose and insulin in VMH-lesioned or sham-operated rats. Half the animals had been adrenodemedullated several weeks previously $(\boldsymbol{\Delta}, \triangle)$. VMH lesions reduced the rise in FFA in both intact $(\bullet)$ and adrenodemedullated rats $(\boldsymbol{\Delta})$. Adrenodemedullation prevented the rise in glucose

VMH-lesioned rats and dropped after the injection of 2-DG (Fig. 4). A similar blunting of the rise in glycerol and NEFA was observed in VMH-lesioned rats after fasting for 8 or $24 \mathrm{~h}$, after a forced swim of $60 \mathrm{~min}$ duration, and after exposure to the cold for $1 \mathrm{~h} \mathrm{[34].}$

The second experiment examined the mobilization of lipids during fasting. Several experimental groups were used including animals with denervation of the retroperitoneal fat pad on one side, those with unilateral VMH or LH lesions, and animals with bilateral VMH or LH lesions [4]. All animals were adrenodemedullated 5 to 6 weeks prior to the study. Prior to initiating the fast, a lumbar sympathecomy was performed under ether anaesthesia. All neural connections on the left side were removed with the aid of a binocular dissecting microscope. The nonsympathetectomized rats had sham-operations. Two days later unilateral VMH-LH lesions were made ipsilaterally, and bilateral lesions were placed in both the VMH and LH areas in a separate group of animals. Fasting continued for 48 or $96 \mathrm{~h}$. At sacrifice the right and left retroperitoneal fat pads and kidneys were removed and weighed. There was no difference in the right and left kidney weights in any of the groups. Animals with denervated fat pads lost fat from the denervated fat pad significantly more slowly than from the intact one (Fig. 5). This is shown at both $48 \mathrm{~h}$ (open bars) and at $96 \mathrm{~h}$ (cross hatched bars). The rats with ipsilateral VMH lesions also mobilized less fat from the retroperitoneal fat pads than the sham-operated groups. The animals with

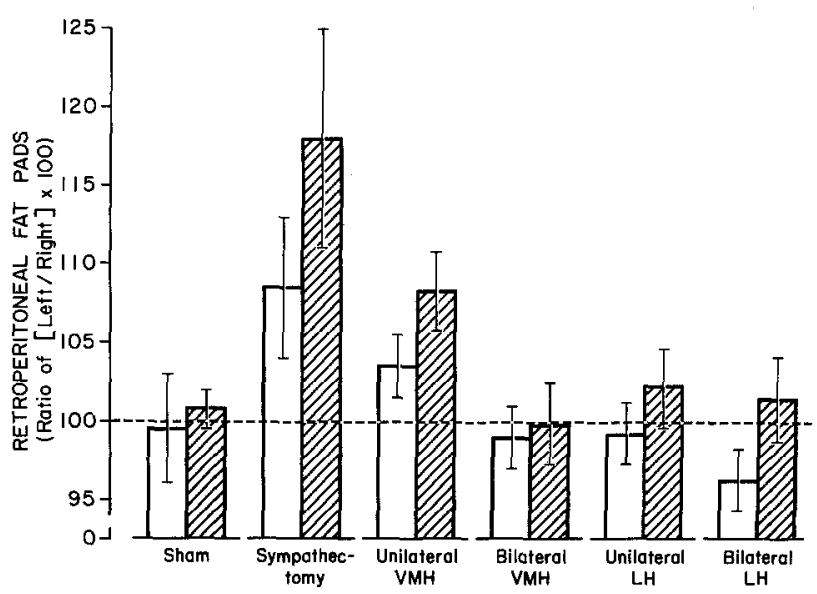

Fig. 5. Effect of unilateral sympathectomy, ipsilateral VMH or LH lesions and bilateral VMH or LH lesions on the weight of the retroperitoneal fat pad. Animals underwent unilateral sympathectomy several days prior to beginning the fast. Unilateral or bilateral lesions in the ventromedial or lateral hypothalamus (VMH or LH) were placed at the initiation of a 48 or $96 \mathrm{~h}$ fast. Weight loss from the retroperitoneal fat pad was reduced on the side of the unilateral sympathectomy and the ipsilateral VMH lesion. Bilateral LH lesions had an effect after 48 hours ( $\square$ ) of fasting, but not at 96 hours $\mathbb{Z}$. Each bar is the mean \pm SEM (0)

LH lesions or bilateral VMH lesions showed no differences. These results provide physiological evidence for a link between the VMH and the ipsilateral sympathetic nervous system, and suggest that destruction of the VMH may impair sympathetic function. 


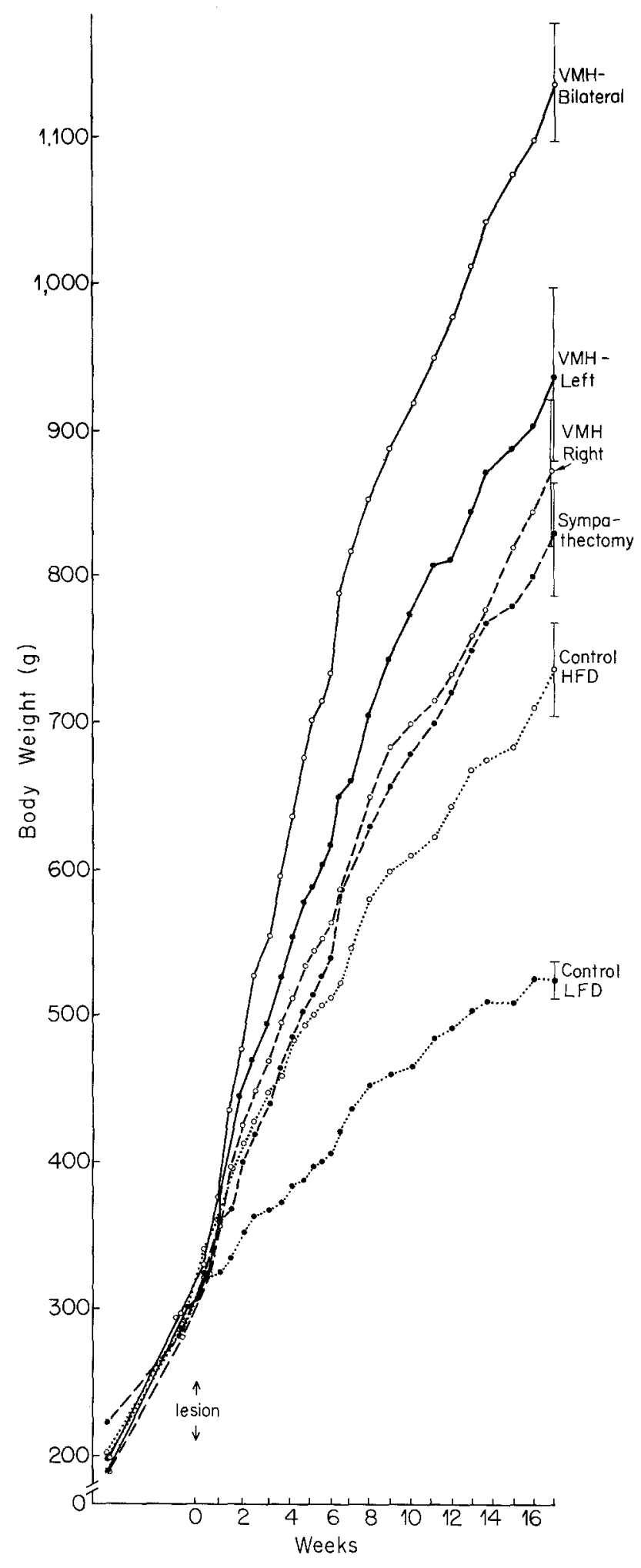

Fig. 6. Effect of sympathectomy and VMH lesions on weight gain in rats fed a high fat diet. The rats with bilateral lesions gained most weight, those on the chow diet the least. The rats with the right or left sided unilateral VMH lesions were slightly heavier than those with unilateral sympathectomy and were in between diet alone and the effects of a bilateral VMH lesion
The obvious corollary to the observation that sympathectomy slowed the weight loss of the retroperitoneal fat pad during fasting was that it might enhance weight gain by slowing fat mobilization during the intermeal period when fat stores are being mobilized for fuel. Since ipsilateral ventromedial hypothalamic lesions mimic the effect of abdominal sympathectomy, the weight gain of rats with unilateral lesions ought to be similar to that of the sympathectomized animal. For this experiment, Osborne Mendel rats were fed either a high fat diet or, in one control group, laboratory chow. The following groups were used: unilateral ventromedial hypothalamic lesions on the right side $(n=10)$, or on the left side $(n=9)$; bilateral ventromedial hypothalamic lesions $(\mathrm{n}=14)$; left sided abdominal sympathectomy $(n=11)$; and sham operations of each group with half sacrificied at 8 weeks and the other half at 16 weeks. The data are summarized in Table 4 and Figure 6 . After 8 weeks the fat fed animals were significantly heavier than the animals fed laboratory chow confirming the often noted sensitivity of the Osborne Mendel to develop obesity when fed a high fat diet. Of interest was that the weight gain after 8 weeks was significantly higher in the sympathectomized animals (171 vs. $225 \mathrm{~g}$ ). This in turn was of similar magnitude to the weight gain of the right sided ( $227 \mathrm{~g}$ ) of left sided ( $295 \mathrm{~g}$ ) VMH lesions. As expected, animals with the bilateral VMH lesions gained the most weight. During the next 8 weeks, weight gains were smaller and no longer significantly different, indicating that they were approaching a plateau. The group sacrificed at 16 weeks showed the same relationships in the fat pad weights for both the epididymal and retroperitoneal areas as observed with the body weights. There were no differences between right and left sides in any of the measurements which were made, except for the testicular weights which were smaller on the left side in the sympathectomized animals. As we have previously noted, the salivary glands of VMH lesioned rats were smaller than in controls.

Unilateral VMH lesions have previously been reported to produce small increments in body weight in animals $[9,25,30,32]$. The present experiments confirm this observation and show that the magnitude of the gain in weight was intermediate between the control and bilaterally lesioned rats when the diet was high in fat. Of particular interest were the observations on the animals with left sided sympathectomy. Their weight gain during the first 8 weeks on the high fat diet was significantly greater than that of the sham-operated animals fed the same diet, but slightly less than in the rats with unilateral lesions (Fig. 6). Although we had anticipated seeing 
Table 4. Effect of unilateral abdominal sympathectomy and VMH-lesions on body weight and organ weights in rats

\begin{tabular}{|c|c|c|c|c|c|c|}
\hline \multirow{3}{*}{$\begin{array}{l}\text { Diet } \\
\text { Operation } \\
\text { Number of animals }\end{array}$} & \multirow{2}{*}{$\begin{array}{l}\text { Chow } \\
\text { Sham }\end{array}$} & \multicolumn{5}{|l|}{ High fat } \\
\hline & & Sham & Sympathectomy & VMH-R & VMH-L & VMH-Bilateral \\
\hline & 7 & 10 & 11 & 10 & 9 & 14 \\
\hline \multicolumn{7}{|l|}{ Body weight $g$} \\
\hline Initial & $311 \pm 8.5$ & $340 \pm 8.5$ & $327 \pm 9.9$ & $312 \pm 10.2$ & $321 \pm 5.0$ & $332 \pm 13$ \\
\hline 8 weeks & $403 \pm 5.5$ & $511 \pm 14.2$ & $552 \pm 18$ & $573 \pm 25.7$ & $617 \pm 22.7$ & $732 \pm 31.8$ \\
\hline$\Delta$ & $92.7 \pm 10.9$ & $171 \pm 9.7$ & $225 \pm 10.4$ & $227 \pm 18.9$ & $295 \pm 20.2$ & $400 \pm 26.2$ \\
\hline 16 weeks & $522.1 \pm 14.2$ & $758 \pm 50$ & $826 \pm 41.0$ & $869 \pm 51.1$ & $937 \pm 60$ & $11134 \pm 39.4$ \\
\hline$\Delta$ & $106.3 \pm 13.3$ & $251 \pm 27.6$ & $245 \pm 15.1$ & $283 \pm 27.4$ & $287 \pm 26$ & $319 \pm 26.3$ \\
\hline Liver $\mathrm{g}$ & $13.3 \pm 0.51$ & $18.4 \pm 1.56$ & $21.8 \pm 1.64$ & $21.4 \pm 1.8$ & $32.0 \pm 3.9$ & $35.1 \pm 2.85$ \\
\hline \multicolumn{7}{|l|}{ Epididymal fat $\mathrm{g}$} \\
\hline $\mathrm{R}$ & $3.79 \pm 0.78$ & $13.5 \pm 1.57$ & $15.9 \pm 1.96$ & $17.9 \pm 2.0$ & $18.4 \pm 1.83$ & $17.7 \pm 1.03$ \\
\hline $\mathbf{L}$ & $3.26 \pm 0.40$ & $12.9 \pm 1.12$ & $15.1 \pm 1.67$ & $17.0 \pm 2.0$ & $17.8 \pm 1.65$ & $17.9 \pm 1.26$ \\
\hline \multicolumn{7}{|l|}{ Retroperitoneal fat $\mathrm{g}$} \\
\hline $\mathrm{R}$ & $4.40 \pm 0.55$ & $21.8 \pm 2.68$ & $28.1 \pm 3.71$ & $33.7 \pm 5.0$ & $38.6 \pm 2.66$ & $49.9 \pm 5.4$ \\
\hline $\mathrm{L}$ & $4.70 \pm 0.68$ & $22.0 \pm 2.8$ & $27.5 \pm 3.83$ & $33.6 \pm 5.2$ & $36.9 \pm 2.91$ & $47.4 \pm 5.2$ \\
\hline \multicolumn{7}{|l|}{ Salivary glands mg } \\
\hline $\mathrm{R}$ & \pm 19.4 & $349 \pm 13.7$ & $371 \pm 30.3$ & $336 \pm 23$ & $365 \pm 16$ & $279 \pm 15$ \\
\hline $\mathrm{L}$ & $359 \pm 24$ & $343 \pm 17.6$ & $415 \pm 16.7$ & $371 \pm 24$ & $364 \pm 10$ & $302 \pm 14$ \\
\hline Lee index & $0.3176 \pm 0.007$ & $0.3556 \pm 0.0161$ & $0.3626 \pm 0.0152$ & $0.3608 \pm 0.0147$ & $0.3827 \pm 0.0057$ & $0.4151 \pm 0.0260$ \\
\hline
\end{tabular}

differences in fat pad weights between the two sides in these animals such differences were not present at either 8 or 16 weeks. This implies that the lipogenesis which occurs in the post-prandial period in the presence of insulin refills both fat pads. Presumably, the mobilization of fat from the denervated pad is slower than in the innervated one because blood flow can be more accurately controlled to supply acceptors for the fatty acids which are mobilized. Whatever the mechanism, the data here showing the similarity between the weight gain of unilateraly VMH lesioned rats and the animals with unilateral abdominal sympathectomy supports the concept that reduced activity of the sympathetic nervous system may be one of the factors involved in the weight gain of these animals $[22,27]$.

In order to explore more thoroughly the relative contribution of each component of the autonomic nervous system to hyperinsulinaemia, we performed an additional experiment. Four weeks after VMH lesions, rats which had been fasted overnight were infused with epinephrine $(1.0 \mu \mathrm{g} / \mathrm{min} / \mathrm{kg})$ to inhibit the effects of sympathetic suppression. Activity of the vagus nerve was inhibited by administration of atropine $(1 \mathrm{mg} / \mathrm{kg} \mathrm{IP}$ and $1 \mathrm{mg} / \mathrm{kg} \mathrm{SC})$. Insulin measurements were done in the fasted state and after glucose administration. Insulin was assayed by the method of Hales and Randle [12]. In the basal or fasted state both epinephrine and atropine decreased serum insulin levels in VMH obese rats (Table 5). After injecting glucose, epinephrine inhibited the
Table 5. Effect of atropine and epinephrine on insulin levels in $\mathrm{VMH}$-lesioned rats

\begin{tabular}{llc}
\hline Treatment & \multicolumn{1}{l}{$\begin{array}{l}\text { Insulin } \\
\mathrm{ng} / \mathrm{ml}\end{array}$} \\
\cline { 2 - 3 } & Basal $^{\mathrm{a}}$ & \multicolumn{2}{c}{ Glucose stimulated } \\
& $4.7 \pm 0.5$ & $16.4 \pm 2.0$ \\
\hline Saline & $2.8 \pm 0.4$ & $4.5 \pm 0.6$ \\
Epinephrine & $1.8 \pm 0.2$ & $8.8 \pm 0.9$ \\
Atropine & $2.0 \pm 0.3$ & $2.8 \pm 0.4$ \\
\hline
\end{tabular}

a Data were obtained 15 minutes after drug administration

b Data were obtained 4 minutes after glucose injection $(0,5 \mathrm{~g} / \mathrm{kg}$, IV) during drug administration

increased insulin level by $90 \%$, but atropine inhibited it only $50 \%$. Epinephrine and atropine together completely blocked the increase of insulin. It was concluded that stimulation of the vagus nerve, and suppression of sympathetic nerves both contribute to the hyperinsulinaemia of VMH lesioned rats.

\section{Interaction of $V M H$ and $L H$ Lesions}

To examine the effect of a VMH lesion introduced into animals whose body weights had been lowered by LH lesions, the following experiment was performed. Animals were conditioned to the laboratory for two weeks while eating laboratory chow and having access to tap water ad libitum. At the end of that time groups of 12 to 13 rats were given lateral 


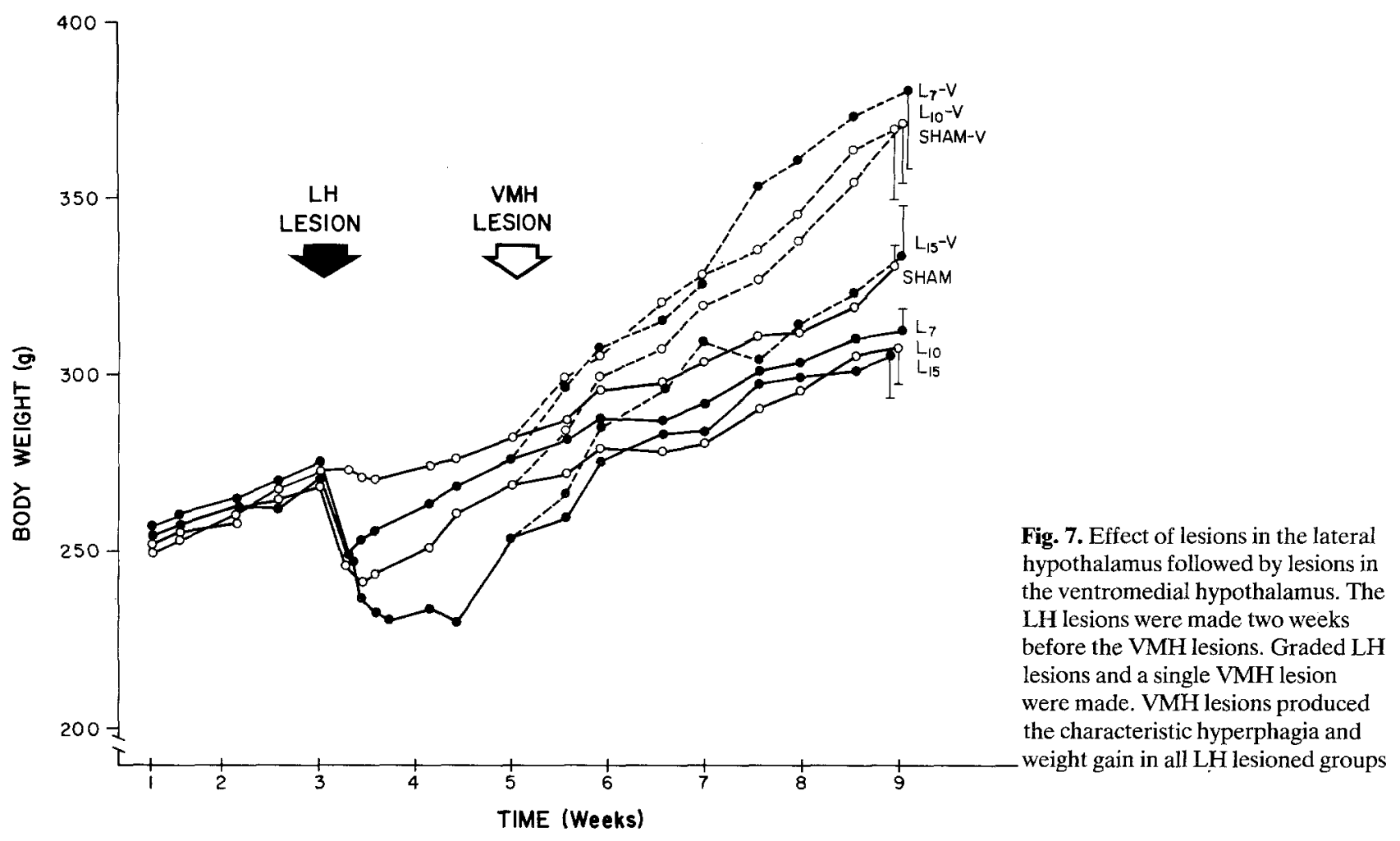

hypothalamic lesions by passing ( $1 \mathrm{mAmp}$ ) anodal current through the tip of an insulated electrode for 7 $(\mathrm{n}=12), 10(\mathrm{n}=12)$, or $15(\mathrm{n}=13)$ seconds. The changes in body weight were greatest for the highest duration of current flow and smaller for the shorter intervals (Fig. 7). The food intake was reduced in all lesioned groups in relation to the duration of the current. Nocturnal food intake declined but there was little or no change during the day time hours. Two weeks after the LH lesion, half of each group received $\mathrm{VMH}$ lesions with a current of $1 \mathrm{mAmp}$ for $15 \mathrm{sec}$ with $\pm 0.7 \mathrm{~mm}$ coordinates from the midlines. There was a rise in body weight in all VMH lesioned groups. The 7 and 10 second LH groups with VMH lesions reached similar weights by 4 weeks after the lesion. The VMH lesioned rats with the largest $\mathrm{LH}$ lesions were only at the level of the sham-operated animals.

The final body weight and several other parameters of function are shown in Tables 6 and 7 . The Lee index was higher in all VMH-lesioned groups indicating that the $\mathrm{VMH}$ lesion had increased body fatness. As reported previously [22] the salivary glands were smaller in the VMH lesioned rats and this occurred in all groups regardless of the size of the $\mathrm{LH}$ lesion. The LH lesion itself, however, did not appear to significantly affect the size of the salivary glands. The smal- ler body weights of the LH lesioned rats was associated with smaller livers and smaller epididymal fat pad weights (Table 6). The concentrations of glucose determined at the time of sacrifice are shown in Table 7 along with concentrations of insulin and glucagon. There was no significant effect on plasma glucose. The insulin levels were higher in all of the VMH lesioned groups except for the sham-VMH sham-LH group. The reason why VMH lesions did not produce higher insulins in this group is unknown. Glucagon levels appeared to be reduced in the LH lesioned rats, but the variance of the glucagon measures makes thes differences not statistically significant.

The similarity of the final body weights after VMH lesions in rats with different sized LH lesions suggests that these two operative procedures act independently. The smaller salivary glands and increases in insulin levels in the VMH lesioned groups suggest that the changes in the autonomic nervous system which have been demonstrated in the VMH-lesioned animals occurred in the presence of the LH lesions. Like a growing body of data, the present experiment provides no evidence for a control of the VMH lesion over the primary food seeking activity of the lateral hypothalamus, but rather suggests that they function independently. 
Table 6. Effect of LH followed by VMH lesions on body and organ weights

\begin{tabular}{lllllll}
\hline $\begin{array}{llll}\text { Lesion } \\
\text { LH }\end{array}$ & VMH & $\begin{array}{l}\text { Final } \\
\text { body wt } \\
\mathrm{g}\end{array}$ & $\begin{array}{l}\text { Lee } \\
\text { index }\end{array}$ & $\begin{array}{l}\text { Liver } \\
\mathrm{g}\end{array}$ & $\begin{array}{l}\text { Salivary } \\
\text { gland } \\
\mathrm{mg}\end{array}$ & $\begin{array}{l}\text { Epididymal } \\
\text { fat pad } \\
\mathrm{mg}\end{array}$ \\
$\mathrm{Sham}$ & Sham & $331 \pm 6.3$ & $310 \pm 5.6$ & $8.75 \pm 0.85$ & $506 \pm 50$ & $5.9 \pm 1.2$ \\
& + & $371 \pm 20.2$ & $322 \pm 3.8$ & $9.37 \pm 1.19$ & $410 \pm 31$ & $12.1 \pm 2.2$ \\
$7 \mathrm{sec}$ & Sham & $313 \pm 10.8$ & $311 \pm 6.2$ & $7.55 \pm 0.70$ & $473 \pm 44$ & $4.9 \pm 0.9$ \\
& + & $381 \pm 22.2$ & $323 \pm 6.7$ & $9.85 \pm 1.32$ & $424 \pm 61$ & $10.6 \pm 2.3$ \\
$10 \mathrm{sec}$ & Sham & $308 \pm 17.3$ & $309 \pm 8.5$ & $7.27 \pm 0.79$ & $462 \pm 46$ & $3.6 \pm 1.2$ \\
& + & $327 \pm 24.1$ & $326 \pm 1.7$ & $9.40 \pm 0.88$ & $382 \pm 36$ & $10.1 \pm 2.0$ \\
$15 \mathrm{sec}$ & Sham & $307 \pm 15.2$ & $310 \pm 4.5$ & $7.38 \pm 0.87$ & $471 \pm 47$ & $4.5 \pm 1.4$ \\
\hline
\end{tabular}

Lee index $=\sqrt[3]{\text { Body Wt }}(\mathrm{g}) /$ Naso-anal length $(\mathrm{cm})$

Table 7. Effect of LH-lesions followed by VMH lesions on some metabolic parameters

\begin{tabular}{lllll}
\hline LH & VMH & $\begin{array}{l}\text { Glucose } \\
\mathrm{mg} / \mathrm{dl}\end{array}$ & $\begin{array}{l}\text { Insulin } \\
\mathrm{ng} / \mathrm{ml}\end{array}$ & $\begin{array}{l}\text { Glucagon } \\
\mathrm{pg} / \mathrm{ml}\end{array}$ \\
\hline Sham & Sham & $100 \pm 1.8$ & $1.3 \pm 0.3$ & $154 \pm 59$ \\
& + & $104 \pm 2.8$ & $1.1 \pm 0.2$ & $133 \pm 44$ \\
& Sham & $96 \pm 1.0$ & $0.9 \pm 0.3$ & $188 \pm 22$ \\
$7 \mathrm{sec}$ & + & $93 \pm 2.7$ & $1.2 \pm 0.2$ & $162 \pm 17$ \\
& + & $99 \pm 2.4$ & $1.2 \pm 0.2$ & $117 \pm 20$ \\
$10 \mathrm{sec}$ & Sham & $104 \pm 4.4$ & $3.5 \pm 1.5$ & 281 \\
& + & $94 \pm 3.4$ & $0.8 \pm 0.2$ & $94 \pm 34$ \\
& Sham & $92 \pm 3.2$ & $1.7 \pm 0.3$ & 201 \\
\hline
\end{tabular}

\section{Hyperinsulinaemia and Hyperphagia}

In the experiment with pancreatic transplantation food intake increased about $70 \%$ after VMH lesions when insulin rose, whereas without hyperinsulinaemia food intake increased by only $20 \%$. This suggests that hyperphagia in VMH lesioned rats is in part due to the hyperinsulinaemia. The mechanism for hyperphagia produced by hyperinsulinaemia is unknown and needs further investigation. Several possibilities can be postulated; 1) Increasing peripheral glucose utilization produced by hyperin- sulinaemia could serve as the signal for food seeking behavior; 2) Glucoprivation in cerebral glucoreceptor cells by insulin could also stimulate food intake [39]; 3) Insulin receptors in the nuclei of the lateral hypothalamus, proposed by Oomura et al., could explain the mechanism of hyperphagia by hyperinsulinaemia [35]. This hypothesis is attractive since insulin receptors have recently been found in the brain [15]; or 4) Finally hyperinsulinaemia may have a close relationship to peptide hormones which stimulate or inhibit food intake such as cholecystokinin or $\beta$-endorphin $[29,40]$.

Increase of food intake without hyperinsulinaemia after VMH lesions also needs further investigation to elucidate the mechanisms of food intake which are related to hypothalamic function.

\section{Conclusion}

This paper develops an Autonomic Hypothesis for explaining many of the metabolic features which occur after VMH lesions [19]. This hypothesis could also explain the mechanisms for development of ventromedial hypothalamic obesity in a framework like that shown in Figure 8.

After VMH lesions, the balance of autonomic nervous system would be changed. This change would suppress some components of the sympathetic nerves and stimulate the vagus. The combined effects would produce hyperinsulinaemia.

The hyperinsulinaemia would increase lipogenesis in liver and adipose tissue. It would also 


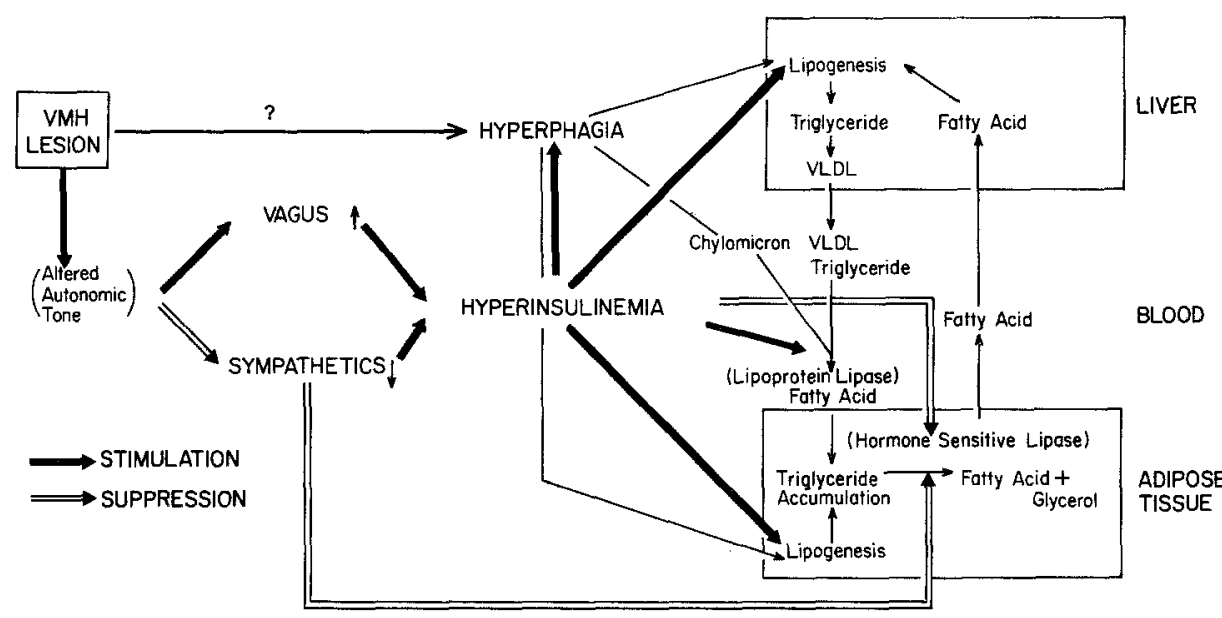

Fig. 8. A schematic mechanism integrating the central nervous system with the peripheral metabolic events in animals with injury to the ventromedial hypothalamus (see text) increase lipoprotein lipase activity and the elevated lipoprotein lipase would accelerate deposition of exogenous (chyromicron) and endogenous (VLDL triglyceride) lipids into adipose tissue. Both hyperinsulinaemia and suppression of the sympathetic nerves would reduce lipolysis.

Hyperphagia which is produced by hyperinsulinaemia would provide excessive food stuffs for lipogenesis and chylomicrons for fat deposition.

All of these effects cooperatively work toward excessive fat accumulation and contribute to the development of hypothalamic obesity.

\section{References}

1. Bernardis LL, Frohman LA (1970) Effect of lesion size in the ventromedial hypothalamus on growth hormone and insulin levels in the weanling rat. Neuroendocrinology $6: 319-328$

2. Berthoud HR, Jeanrenaud B (1979) Acute hyperinsulinemia and its reversal by vagotomy after lesions of the ventromedial hypothalamus in anesthetized rats. Endocrinology 105: 146-151

3. Bray GA, Gallagher Jr TF (1975) Manifestations of hypothalamic obesity in man: A comprehensive investigation of eight patients and a review of the literature. Medicine (Baltimore) 54: 301-330

4. Bray GA, Nishizawa Y (1978) Ventromedial hypothalamus modulated fat mobilization during fasting. Nature 274: 900-902

5. Bray GA, York DA (1979) Hypothalamic and genetic obesity in experimental animals: An autonomic and endocrine hypothesis. Physiol Rev 59: 719-809

6. Brobeck JR (1946) Mechanism of development of obesity in animals with hypothalamic lesions. Physiol Rev 26: 541-559

7. Brobeck JR (1960) Food and temperature. Recent Prog Horm Res 16: 439-466

8. Frohman LA, Bernardis LL (1968) Growth hormone and insulin levels in weanling rats with ventromedial hypothalamic lesions. Endocrinology 82: 1125-1132

9. Gold RM, Quackenbush PM, Kapatos G (1972) Obesity fol- lowing combination of rostrolateral to $\mathrm{VMH}$ cut and centralateral mamillary area lesion. J Comp Physiol Psychol 79: 210-218

10. Goldman JK, Schnatz JD, Bernardis LL, Frohman LA (1972) Effects of ventromedial hypothalamic destruction in rats with pre-existing streptozotocin induced diabetes. Metabolism 21: 132-136

11. Hales CN, Kennedy GG (1964) Plasma glucose, non-esterified fatty acids and insulin concentrations in hypothalamic-hyperphagic rats. Biochem J 90: 620-624

12. Hales CN, Randley PJ (1963) Immunoassay of insulin with insulin antibody precipitate. Biochem J 88: 137-146

13. Han PW, Frohman LA (1970) Hyperinsulinemia in tube-fed hypophysectomized rats bearing hypothalamic lesions. Am J Physiol 219: 1632-1636

14. Han PW, Liu AC (1966) Obesity and impaired growth of rats force fed 40 days after hypothalamic lesions. Am J Physiol 211: $229-231$

15. Havrankova J, Roth J, Brownstein M (1978) Insulin receptors are widely distributed in the cerebral nervous system of the rats. Nature 272: $827-829$

16. Hetherington A, Ranson SW (1940) Hypothalamic lesions and adiposity in the rat. Anat Rec 78: 149-172

17. Hustvedt BE, Løvø A (1972) Correlation between hyperinsulinemia and hyperphagia in rats with ventromedial hypothalamic lesions. Acta Physiol Scand 84: 29-33

18. Inoue S, Bray GA (1977) The effects of subdiaphragmatic vagotomy in rats with ventromedial hypothalamic obesity. Endocrinology 100: 108-114

19. Inoue S, Bray GA (1979) An autonomic hypothesis for hypothalamic obesity. Life Sci 25: 561-566

20. Inoue S, Bray GA, Mullen YS (1977) Effect of transplantation of pancreas on development of hypothalamic obesity. Nature 266: 742-744

21. Inoue S, Bray GA, Mullen YS (1978) Transplantation of pancreatic $\beta$-cells prevents development of hypothalamic obesity in rats. Am J Physiol 235: E266-E271

22. Inoue S, Campfield LA, Bray GA (1977) Comparison of metabolic alterations in hypothalamic and high fat dietinduced obesity. Am J Physiol 233: R162-R168

23. Inoue S, Bray GA (1980) Role of the autonomic nervous system in the development of ventromedial hypothalamic obesity. Brain Res Bull 5 (Suppl 4): 119-125

24. Jungas RL, Ball EG (1963) Studies on the metabolism of adipose tissue. XII. The effects of insulin and epinephrine on free 
fatty acid and glycerol production in the presence and absence of glucose. Biochemistry 2: 383-388

25. Kapatos G, Gold RM (1973) Evidence for ascending noradrenergic mediation of hypothalamic hyperphagia. Pharmacol Biochem Behav 1: 81-87

26. Kanarek RB, Hirsch E (1977) Dietary-induced over-eating in experimental animals. Fed Proc 36: 154-158

27. Kasemasri S, Bernardis LL, Schnatz JD (1972) Fat mobilization in adipose tissue of weanling rats with hypothalamic obesity. Horm Res 3: 97-104

28. Lockhart-Ewart RB, Mok C, Martin JM (1976) Neuroendocrine control of insulin secretion. Diabetes 25: 96-100

29. Margules DL, Moisset B, Lewis MJ, Shibuya H, Pert CB (1978) $\beta$-endorphin is associated with overeating in genetically obese mice (ob/ob) and rats (fa/fa). Science 202: 988-991

30. Marshall JF (1975) Increased orientation to sensory stimuli following medial hypothalamic damage in rats. Brain Res 86 : 373-387

31. Martin JM, Konijnendijk W, Bouman PR (1974) Insulin and growth take off hormone secretion in rats with ventromedial hypothalamic lesions maintained on restricted food intake. Diabetes 23: 203-208

32. Mayer J, Barnett RJ (1955) Obesity following unilateral hypothalamic lesions in rat. Science 121: 599-600

33. Moltz JH, Dobbs RE, McCann SM, Fawcett CP (1977) Effects of hypothalamic factors on insulin and glucagon release from the islets of Langerhans. Endocrinology 101: 196-202

34. Nishizawa Y, Bray GA (1978) Ventromedial hypothalamic lesions and the mobilization of fatty acids. $J$ Clin Invest 61 : 714-721

35. Oomura Y, Sugimori M, Nakamura T, Yamada (1975) Contribution of electrophysiological techniques to the understanding of central control systems. In: Mogenson GJ, Calaresu FR (eds) Neural integration of physiological mechanisms and behavior. University of Toronto Press, Toronto, p 375-395

36. Powley TL, Opsahl CA (1974) Ventromedial hypothalamic obesity abolished by subdiaphragmatic vagotomy. Am J Physiol 226: 25-33

37. Ridley PT, Brooks FP (1965) Alterations in gastric secretion following hypothalamic lesions producing hyperphagia. Am J Physiol 209: 319-323

38. Rohner R, Dufour AC, Karakash C, Marchand Y, Rud KB, Jeanrenaud B (1977) Immediate effect of lesion of the ventromedial hypothalamic area upon glucose-induced insulin secretion in anaesthetized rats. Diabetologia 13: 239-242

39. Smith G, Epstein A (1969) Increased feeding in response to decreased utilization in the rat and monkey. Am J Physiol 217: 1083-1087

40. Straus E, Yalow RS (1979) Cholecystokinin in the brains of obese and non obese mice. Science 203: 68-69

41. Weber CJ, Hardy MA, Lerner RL, Felig P, Reemtsma K (1976) Hyperinsulinemia and hyperglucagonemia following pancreatic islet transplantation in diabetic rats. Diabetes 25: 944-948

42. Woods SC, Porte Jr D, (1974) Neural control of the endocrine pancreas. Physiol Rev 54: 596-619

43. York DA, Bray GA (1972) Dependence of hypothalamic obesity on insulin the pituitary and the adrenal gland. Endocrinology 90: 885-894

George A. Bray, M. D.

Department of Medicine, Bldg. A-17 Annex

Harbor UCLA Medical School

$100 \mathrm{~W}$. Carson Street

Torrance, CA 90509

USA

\section{Discussion after Bray's Presentation}

Nicolaidis: I am interested in knowing whether or not your rats have certain reflexes which are sometimes seen clinically in patients with autonomic disturbances. For example, some patients with elevated parasympathetic tone have an exaggerated oculo-cardiac reflex such that pushing on their eye causes the heart to slow markedly. These same patients often also have ulcers. Also, do any of the symptoms you observe in your lesioned rats exist in these patients?

Bray: We have never looked for these reflexes in our patients, since most patients with the VMH syndrome usually have brain tumors which have destroyed or distorted the brain in such a way that it would be hard to draw conclusions.

Steffens: Are the plasma epinephrine and nor-epinephrine levels decreased in your $\mathrm{VMH}$-lesioned rats as your theory would predict?

Bray: As far as I know, that experiment hasn't been done.

Porte: It's important to remember that nor-epinephrine levels are probably related more to vascular than to metabolic events, so they might not be expected to be low. A differential change of epinephrine relative to norepinephrine might be more meaningful. I would like to know if there was any problem with your islet-transplanted rats in that the insulin was not secreted into the portal circulation.

Bray: Circulating insulin levels were slightly higher in the non-lesioned, islet-transplanted rats than in their controls.

Powley: I'm concerned about your conclusions as to the importance of the sympathetic nervous system in the VMH syndrome. As I've said before, I believe that the vagus alone can account for the bulk of the syndrome. Jim Cox and I have recently completed an experiment to test this. In order to control for any non-specific effects of vagotomy on food intake, all the rats had gastric catheters. The rats were run in blocks such that a normal, control rat in each block could press a lever for food and eat on a normal schedule. Every time it pressed the lever, all of the other rats in that block were pair-fed intragastrically. In this way, they all received a normal amount of food with a normal meal patterning. Some of these pair-fed rats had vagotomies, some VMH lesions, some both, and so on. Rats with a prior vagotomy, if it was complete, developed no obesity over the 6 weeks following a VMH lesion. Animals with their vagus intact which received a VMH lesion, even with the pair-feeding, became obese and hyperinsulinaemic. If the 
vagotomy was incomplete, some obesity developed. This experiment, with its tight control on food intake, therefore suggests that a disturbance of the sympathetic nervous system cannot play much of a role in the obesity of the $\mathrm{VMH}$ syndrome.

Bray: What you say has considerable merit. It may actually be that the obesity and other components of the syndrome caused by elevated insulin may be entirely due to vagal hyperactivity. Other effects of the lesion, such as gluconeogenesis, however, may be caused by altered sympathetic tone. What we want to suggest is similar to the argument made by Dr. Shimazu, namely that these brain areas control a number of peripheral metabolic responses and that lesions would be expected to disrupt or alter a number of them in diverse ways.

Goldman: Dr. Powley, did you state that the vagotomy reduced the defect in the nasoanal length of $\mathrm{VMH}$-lesioned rats?

Powley: Yes, the tendency existed. Remember that these rats received a fixed amount of calories each day for a month and had to do something with them. We found no evidence for malabsorption and those animals which didn't get fat were trivially longer than those which did. The difference didn't quite reach statistical significance, but the trend was there.
Nicolaidis: Whenever parasympathetic tone is increased, one would expect a relative decrease of sympathetic effects even if there is no change in the CNS sympathetic output. Is what you have seen due to this, or is it more than this?

Bray: The answer probably depends upon the response you are measuring. In a response like lipolysis where the vagus probably plays little role, decreased sympathetic output is probably the major event. But you are correct in that for those responses which are dually controlled by the autonomic nervous system, it would be hard to separate the two possibilities. For example, both effects might well contribute to the change of insulin secretion.

Powley: But this explanation still has a problem because the elevated insulin could also play a role in these other responses such as lipolysis. You cannot say that the sympathetic nervous system is important in the VMH-lesioned animal until you can measure the same response in the absence of hyperinsulinaemia, and you haven't done that.

Sclafani: Do you have to give your rats a high fat diet in order to see the effects on the sympathetic nervouls system?

Bray: I can't answer that. All of our rats were given a high fat diet because we wanted them to get fat faster.

Sclafani: In a quick and dirty experiment, we recently found no difference in salivary weight of knife-cut animals. 